\title{
The power of VNA-driven quasi-optics to sense group molecular action in condensed phase systems
}

\author{
Rob Donnan ${ }^{*}$, Kun Viviana Tian ${ }^{\ddagger}$, Bin Yang ${ }^{\ddagger}$ and Gregory A. Chass ${ }^{\S}$ \\ *School of Electronic Engineering and Computer Science, Queen Mary University of London, \\ Mile End Road, London E1 4NS, United Kingdom, Email: r.donnan@qmul.ac.uk \\ ${ }^{\ddagger}$ Materials Science Research Institute, Department of Oral Diagnostics, Faculty of Dentistry, \\ Semmelweis University, Budapest 1088, Hungary \\ * Engineering, Sports \& Sciences Academic Group, University of Bolton, \\ Bolton, BL3 5AB, United Kingdom \\ ${ }^{\S}$ School of Chemical and Biological Sciences, Queen Mary University of London, \\ Mile End Road, London E1 4NS, United Kingdom
}

\begin{abstract}
The versatility for quasi-optical circuits, driven by modern vector network analysers, is demonstrated for the purpose of low energy $(\mathrm{meV})$ coherent spectroscopy. One such example is shown applied to the curing dynamics of a nonmercury-based dental cement. This highlights the special place the methodology holds as a 'soft-probe' to reveal the timeresolved energetics of condensed phased systems as they selforganise to adopt their low energy state.
\end{abstract}

Index Terms - Quasi-optical, vector network analyser, timeresolved spectroscopy.

\section{INTRODUCTION}

Terahertz methods are being evolved to uniquely enable the spectroscopy of complex organic polymers in their native solvated state. As a 'soft' probe, in contrast to ballistic methodologies such as x-ray and neutron scattering, terahertz ( $\mathrm{THz}$ ) spectroscopy is sensitive to the low energetics (meV) of collective or group modes of action inherent to organic polymer systems, and such modes are increasingly being appreciated to play a fundamental role in the organisation and function of such systems. The ability to study organic molecular dynamics in their water-world is hugely challenging. For example, not all proteins can be crystalised for x-ray diffraction analysis (XRD) and, extrapolating such $\mathrm{XRD}$ analysis to understanding the dissolved protein, does not always easily follow. The $\mathrm{THz}$ method here consists of a vector network analyser (VNA)-driven quasi-optical (QO) circuit. It is stressed that the two together (VNA and QOs) are what is special for this low-energy spectroscopy. Modern VNAs are powerful signal-processing computers supporting heterodyne transceivers that are inherently ultra phase-stable in performance with outstanding spectral resolution to $1 \mathrm{~Hz}$. QOs enable near frequency-independent beam transport at very high efficiencies. Thus, VNA-QOs enables very high control of systematic and random errors - of key importance for tracing a metrology for experimental bio-physics.

Herein we detail two short case studies where the same generic QO circuit is applied to the study of $\mathrm{THz}$ field absorption by 1) the curing characteristic of non-mercurybased, glass-ionomer dental cement, and 2) dissolved proteins. Before treating each case in-turn, the next section outlines the common apparatus of VNA and QOs.

\section{VNA-DRIVEN QUASI OPTICS}

The VNA and associated QO circuit used is depicted in Fig. 1a. Fig. 1b depicts the associated Gaussian-beam tube at the $98 \%$ (green) power-level envelope [1]. In this paper, both exempla studies are investigated by the set-up of this VNAQO combination (Fig.1).

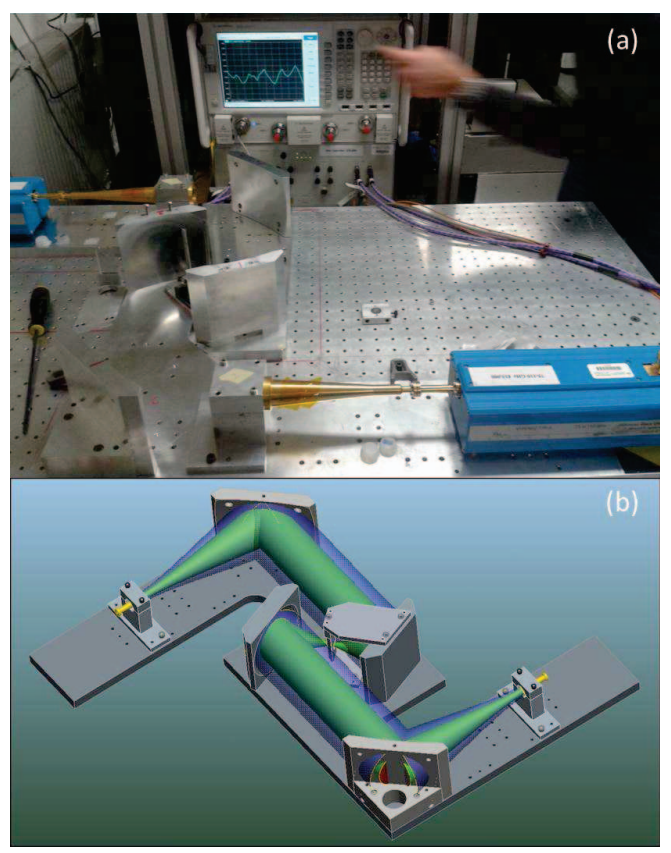

Fig. 1: (a) In the background, with the operator, is the vector network analyser (Agilent, 4-port, PNAX (N5244A)). It connects to the blue transmit and receive frequency-multiplier modules sat on the quasi- 
optical bench (foreground). The intermediate quasi-optical circuit consists of transmit and receive corrugated horns, screw-fitted to their respective multiplier module, together with a mirror-train between them. The inner-most fast-mirror pair controls a broad-band, high-quality focus from beams passed to them from collimating ellipsoids. This is depicted in (b) showing the 98\% (green-tube) Gaussian-beam power envelope.

\section{EXPERIMENTAL}

\section{A. Time-resolved spectroscopy of a glass-ionomer cement reaction.}

The United Nations Environmental Programme's (UNEP) determination to phase out the use of mercury in dental practice has highlighted the use of alternative dental glass cements for filling cavities [2]. Rational characterisation and design of these cements to achieve fracture toughness required for load-bearing service continued to provide challenge. In light of this, VNA-QO sub-THz spectroscopy experiments have been conducted for the $1^{\text {st }}$ time to characterise the setting reaction of these bio-cements [Fig. 2].
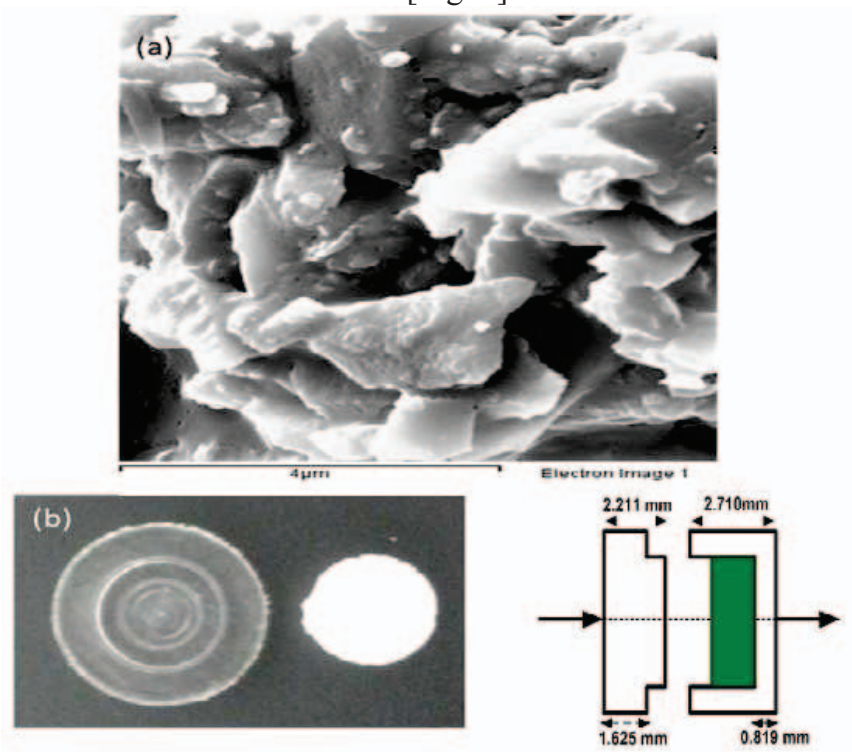

Fig 2: (a) SEM image of cement glass particles showing heterogeneous nature. (b) (left) Real TPX ${ }^{\mathbb{B}}$ cap and glass-ionomer cement (GIC) compress; (right) the schematic plot of TPX ${ }^{\circledR}$ cap and the compress sample-under-test (green area).

To make these measurements relevant to current dental practice, commercial G338 glass (Fig. 2a) and Chemflex liquid have been used, employing standard clinical preparatory mixing. The sample is sandwiched in an $11 \mathrm{~mm}$ diameter TPX ${ }^{\circledR}$ cap and hermetically-sealed with vacuum grease as shown in Fig. 2b.

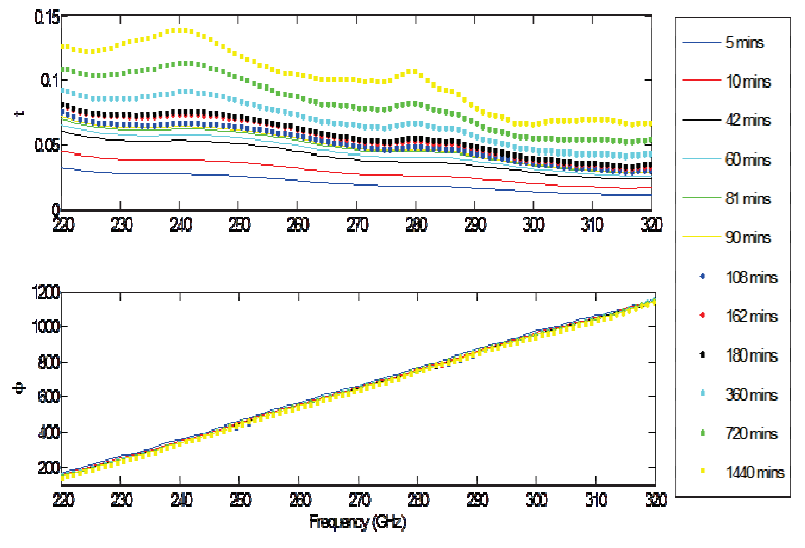

Fig 3: The measured complex transmission spectra of the cement at the selected times (in minutes): (top) the transmission amplitudes and (bottom) the transmission phase information.
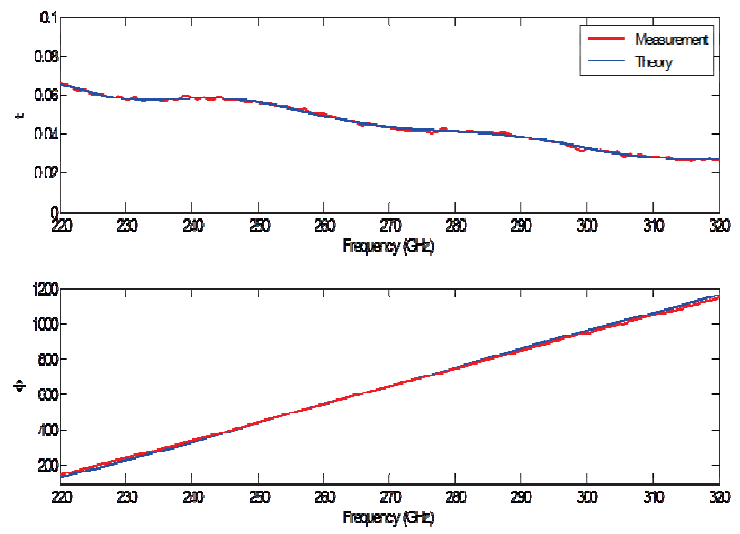

Fig 4: Comparison of the measured and calculated complex transmission spectra of the cement at 60 minutes: (top) the transmission amplitudes and (bottom) the transmission phase information.

The setting reaction was measured continuously for 30 hours from the start of mixing, while complex transmission spectra was sampled at 3 minute intervals for capturing the timedependent THz features.. Selected spectra at 5, 10, 42, 60, 81, $90,108,162,180,360,720$ and 1440 minutes (post-mixing) are plotted in Fig. 3. For the stacked structure consisting of TPX|plate|cement|TPX plate, analysis proceeds using a transfer $\mathrm{T}$ matrix (or ABCD matrix), descriptive of the complex transmission. An iterative method is used to estimate the unknown dielectric constant (i.e spectral complex permittivity) of the cement - by minimising the error between the ABCD-model and experimental data [3,4]. In Fig. 4, a typical theoretically fitted transmission spectrum of the cement is co-plotted together with the measured transmission data. The acquired dielectric permittivity is shown in Fig.5. 

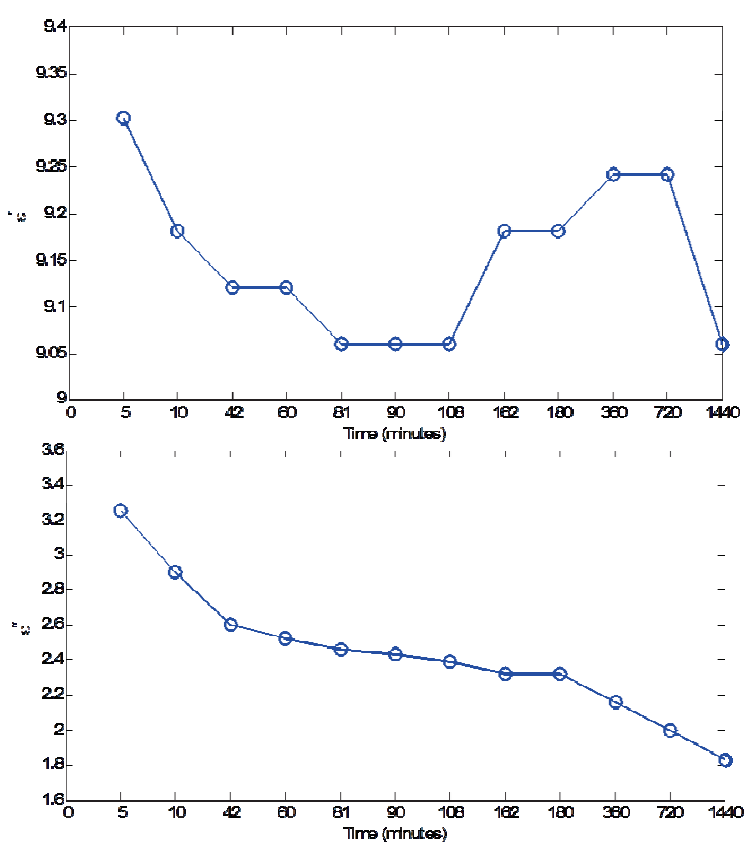

Fig 5: Calculated dielectric constants of the cement: (top) real part of permittivity and, (bottom) imaginary part of permittivity at selected times in minutes.

The setting mechanism is complex due to the multifarious nature of the amorphous phase-separated fluoro-aluminosilicate glass component. The initial setting mechanism is an acid-base reaction between the aqueous poly(acrylic acid) (PAA) and the glass component. The collective THz dynamics at the hybrid interface can be visualised as the twisting, rocking, flexing and compressing of a chaotic flexural pivot centred on Al(IV) [5]. By using all the calculated complex permittivity values, we observe that initially the permittivity falls due to the release of $\mathrm{Ca}^{2+}$ and then recovers close to the initial point where $\mathrm{Al}^{3+}$ emerges [5]. Accordingly, the minimum at 3 hours defines a coupling point in the reaction setting mechanism after the cement has lost its initial plasticity. The cooperative dynamics at the polymer-glass interface promote $\mathrm{Al}^{3+}$ - polymer chelation but will also be susceptible to changes in interfacial stress [5].

\section{CONCLUSION}

The capacity for, and versatility of, vector-networkanalysers in driving quasi-optical circuits for performing timeresolved high-resolution, frequency spectroscopy, has been exemplified in initial work in studying the reaction of alumino-silicate glasses with acrylic acid over a 30 hour setting-reaction time. Dielectric studies form part of a comprehensive mechanical-dynamics study that for the $1^{\text {st }}$ time will guide novel engineering of such bio-cements to have optimum fracture toughness in conjunction with strength.

\section{ACKNOWLEDGEMENT}

The authors would like to thank the Engineering and Physical Sciences Research Council (EPSRC, UK) for generous support (EP/1014845).

\section{REFERENCES}

[1] Y Wang, B Yang, Y Tian, RS Donnan, MJ Lancaster IEEE Transactions on Terahertz Science and Technology 4 (2), 247-253.

[2] United Nations Environment Programme (UNEP) (2013), The Global Mercury Assessment 2013: Sources, Emissions, Releases and Environmental Transport. UNEP Chemicals Branch, Geneva, Switzerland.

[3] Bin Yang, Richard. J. Wylde, Derek. H. Martin, Philippe.Goy, Robert.S.Donnan and Sylvain, Determination of the gyrotropic Characteristics of Hexaferrite Ceramics from 75 to $600 \mathrm{GHz}$ ", IEEE Trans.on Microw. Theory Tech, Vol 58, No.12, Dec, 3587-3597, (2010) .

[4] X. Liu, H. Chen, Bin Yang, X.Chen, Clive Parini and Dongsheng Wen, "Dielectric Property Measurement of Gold Nanoparticle Dispersions in the Millimeter Wave Range", Journal of Infrared and Terahertz Waves, Feb, vol 34, Issue 2, pp 140-151 (2013).

[5] Data submitted for publication by the present authors. 